\title{
Validation of the methylene blue test for assessment of viability of protoscolices from hydatid cysts
}

\author{
Elsayed E. Elowni*, Mohamed Fadl Ahmad, Ghada H. Abdelnabi and Reem M. Badawi \\ Department of Parasitology, Faculty of Veterinary Medicine, University of Khartoum, Shambat, Sudan
}

\begin{abstract}
Background: Methylene blue was used as a vital stain for the assessment of viability of protoscolices from hydatid cysts taking advantage of the chemical nature of the dye as a redox indicator and the kinetically distinct molecular transfer systems of Echinococcus protoscolex for uptake of materials across the tegument.

Aim: The present study attempts to validate the application of methylene blue staining for assessment of viability of protoscolices.

Methods: To validate the criteria by which viability is assessed, control tests were performed using normal protoscolices and protoscolices previously treated with distilled water at $60^{\circ} \mathrm{C}$ for 5 minutes. Performance of methylene blue was further studied at intervals over a period of 50 minutes after protoscolex exposure using $1 \%$ dye concentration.

Results: Normal protoscolices were able to adsorb and reduce the dye and have, therefore, lost the blue color. Protoscolices previously treated with warm water on the other hand, being functionally dead, failed to reduce the adsorbed dye and permanently retained the blue color. Results also indicated that a clear distinction between dead and alive protoscolices can be made within 1 minute. Reading of the test after 10 minutes would be misleading giving a false result.

Conclusion: These findings suggest that viability of protoscolices can be assessed on the basis of acquisition and loss/ retaining of the dye blue color. Increasing the concentration of methylene blue to $1 \%$ was noticed to be associated with remarkable enhancement of contractility, sucker movement, and evagination. Such an excitatory action of the dye may be exploited in viability tests which adopt these criteria.
\end{abstract}

Keywords: Methylene blue, Oxygen, Protoscolices, Viability.

\section{Introduction}

Cystic echinococcosis (hydatidosis) is a serious zoonotic disease of cosmopolitan distribution. It is caused by the infection with hydatid cysts, the larvae of the canine tapeworm Echinococcus granulosus senso lato (s.l.). The cysts develop in a wide range of mammalian hosts, including domestic and wild ruminants, equines as well as humans. A fertile cyst normally contains thousands of invaginated protoscolices which are infective to the definitive hosts, mainly dogs, when they ingest viscera containing the cyst. There is no tangible measure, however, to assess the degree of this infectivity apart from feeding experimentally to the definitive host (dogs), a known number of protoscolices and counting at necropsy, the number of worms that develop in the intestine. This approach, however, though technically feasible, has several limitations not least among which is the difficulty in finding such very small worms, typically $2-7 \mathrm{~mm}$ in length, in the intestine and the potential health hazard of exposure to infective eggs released by the patent worms. To avoid such problems, in vitro staining techniques have been used for assessment of viability of protoscolices (defined as the capacity of being alive) using stains, such as eosin, methylene blue, Giemsa, and others (Esfandiari and Youssef, 2010; Miman et al., 2010).

Methylene blue $\left(\mathrm{C}_{16} \mathrm{H}_{18} \mathrm{ClN}_{3} \mathrm{~S}\right)$, also known as methylthioninium chloride, is a synthetic basic dye. It has wide applications as a dye, as a medication, in aquaculture and in a number of different staining procedures. Methylene blue is also widely used as a redox indicator in analytical chemistry and in biology as an indicator to determine whether eukaryotic cells, such as yeast, are alive or dead. The dye has the property of crossing cell membrane.

The present study attempts to validate the application of methylene blue staining for assessment of viability of protoscolices taking advantage of the chemical nature of the dye as a redox indicator and the kinetically distinct molecular transfer systems of Echinococcus protoscolex for uptake of materials across the tegument (Jeffs et al., 1987). It is proposed that the effective assessment of protoscolex viability may lead to a better understanding of the relative importance of different species of intermediate hosts in parasite transmission and may as well be useful in experimental studies, such as drug evaluation, immunological tests, or in vitro parasite culture. 


\section{Materials and Methods}

\section{Protoscolices}

Hydatid cysts were obtained from the lungs of naturally infected camels slaughtered in Tambool, Gezeira State in Central Sudan, where hydatidosis (echinococcosis) is known to be endemic (Saad and Magzoub, 1986; Elmahdi et al., 2004; Osman and Abdalla, 2013). Cysts were transported in situ to the parasitology laboratory, Faculty of Veterinary Medicine, University of Khartoum, within 7-8 hours after slaughter and were kept in a refrigerator before use the next day. According to Ismail and Saad (2017), protoscolices derived from hydatid cysts of camel origin can remain viable in cystic fluid for 20,7 , and 5 days when kept at $4^{\circ} \mathrm{C}, 25^{\circ} \mathrm{C}$, or $37^{\circ} \mathrm{C}$ constant temperatures, respectively. Cysts were grossly examined. Those with a tender texture apparently containing fluid were dissected free from the surrounding tissue, washed thoroughly from detritus, and slit open in wide-mouth glass containers. Fluid collected from individual cysts was divided into $10-\mathrm{ml}$ tubes and centrifuged at 2,000 rpm for 5 minutes for any protoscolices present to sediment. The supernatant was discarded leaving just a trickle of fluid at the bottom of the tube. Presence of protoscolices in the sediment was confirmed by microscopic examination of a thin smear on a slide.

\section{Staining}

Following centrifugation, a sample of protoscolex sediment was transferred to a glass slide and few drops of $0.1 \%$ or $1 \%$ aqueous methylene blue were added. The sample was covered with cover glass slip and examined microscopically for assessment of viability. To validate the criteria by which viability is assessed, control tests were performed using protoscolices previously treated with distilled water at $60^{\circ} \mathrm{C}$ for 5 minutes and treated with methylene blue According to Moazeni and Alipour-Chaharmahali (2011), treatment with warm water at $50^{\circ} \mathrm{C}, 55^{\circ} \mathrm{C}$, or $60^{\circ} \mathrm{C}$ for 5,2 , or 1 minute, respectively, is $100 \%$ lethal to protoscolices. Effect of the dye on protoscolices was subsequently monitored

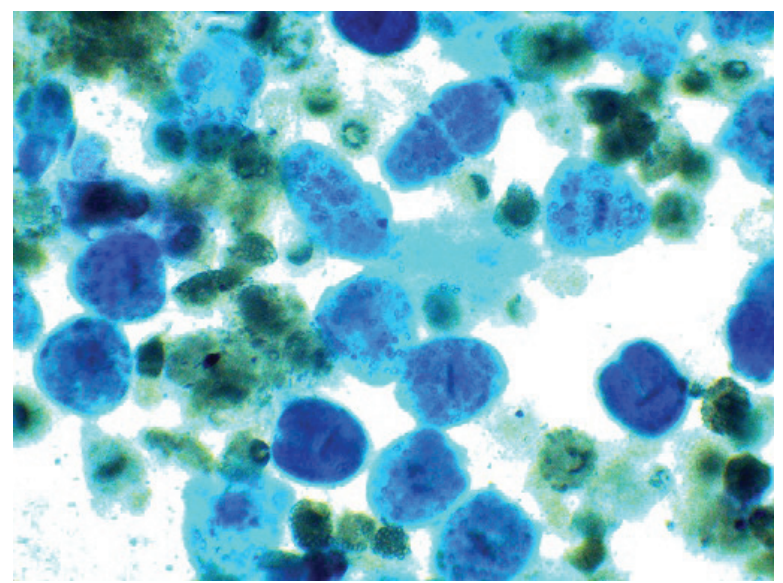

Fig. 1. Uptake of methylene blue $0.1 \%$ by protoscolices previously treated with warm water (Magnification 100×). at interval at $1,5,10,20,25,30,40$, and 50 minutes following dye application taking acquisition/loss of the blue color as criteria for assessment of viability.

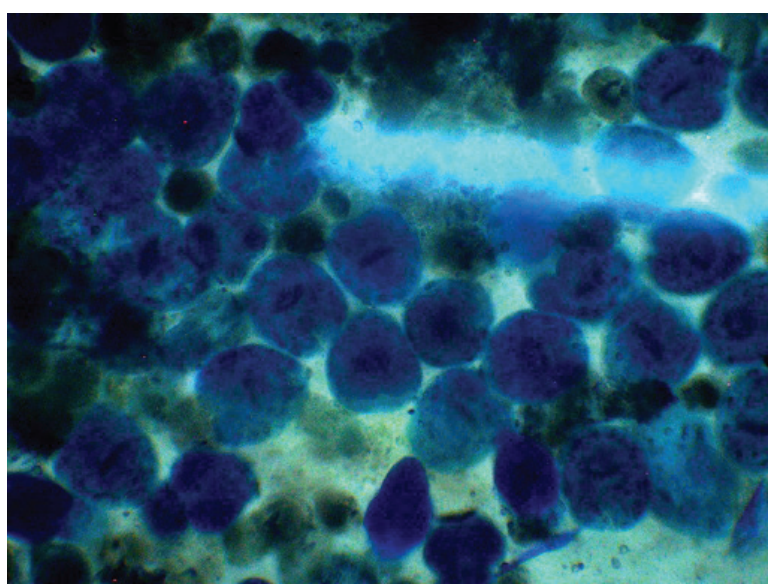

Fig. 2. Uptake of methylene blue $1 \%$ by protoscolices previously treated with warm water (Magnification 100×).

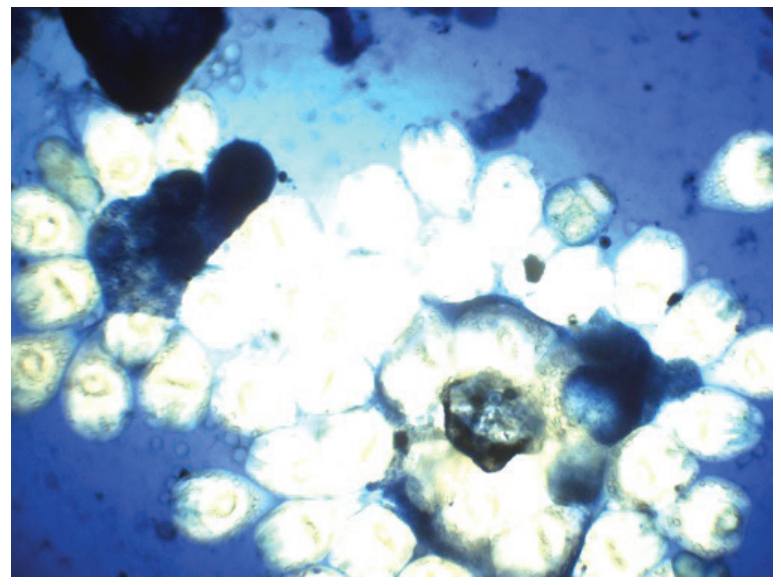

Fig. 3. Reduction of methylene blue $0.1 \%$ by normal protoscolices (Magnification 100×).

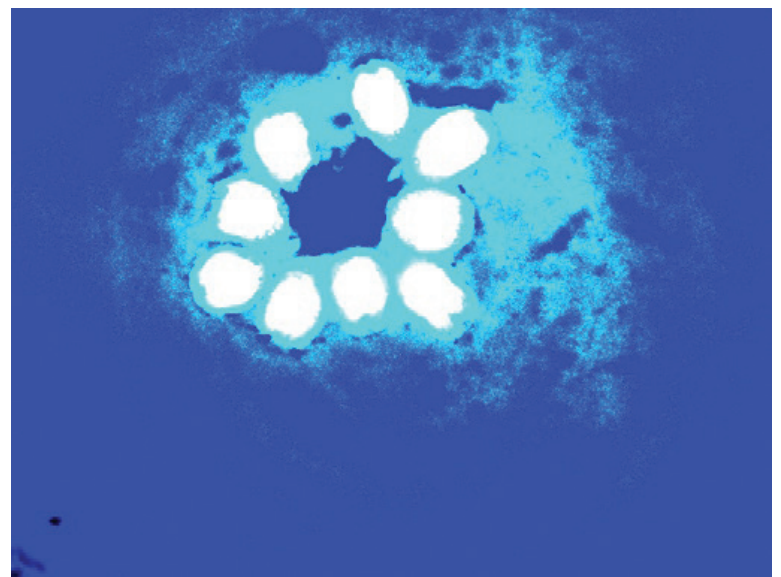

Fig. 4. Reduction of methylene blue $1 \%$ by normal protoscolices (Magnification 100×). 

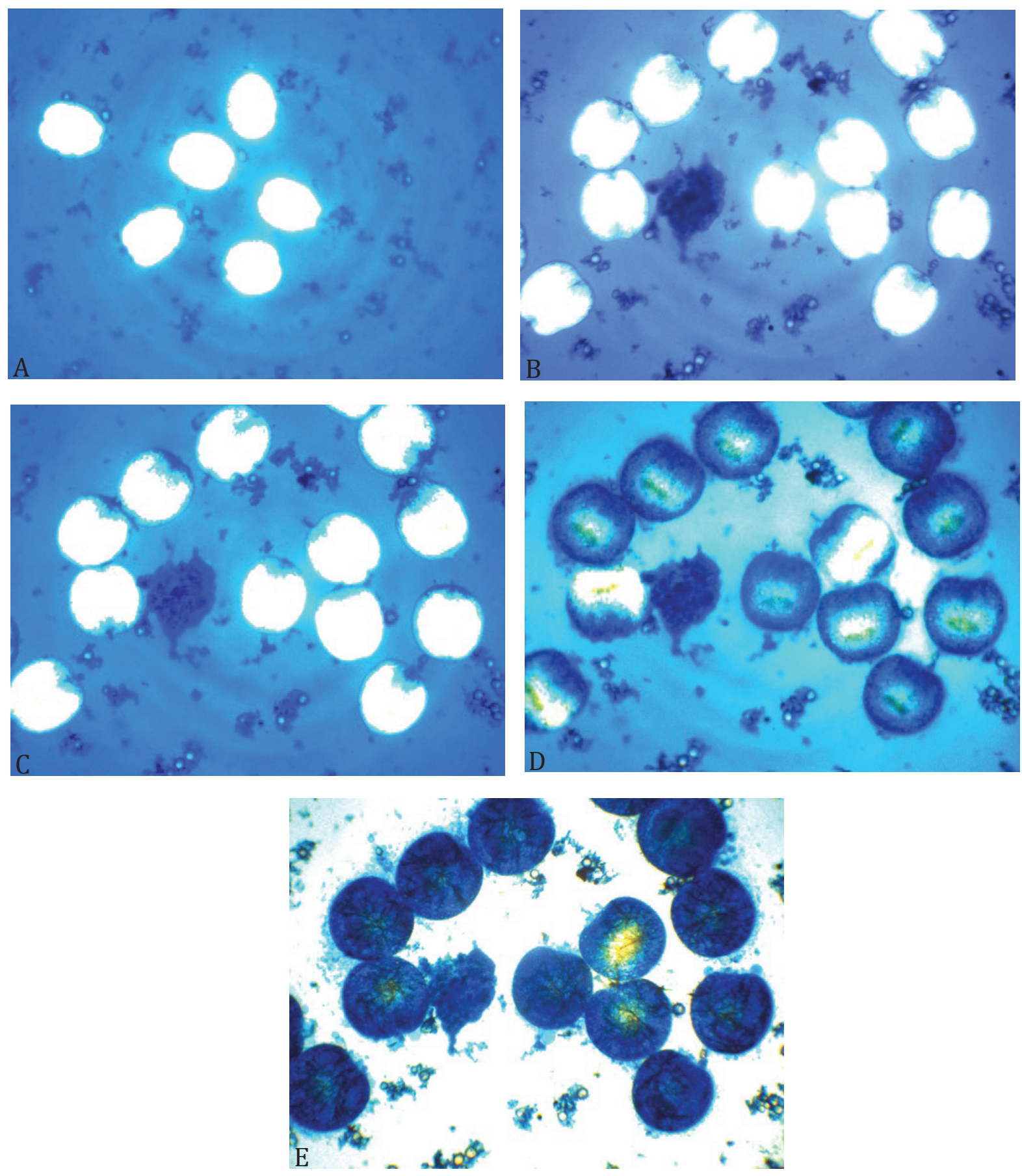

Fig. 5. Progressive uptake of methylene blue 1\% by normal protoscolices at (A) 1 minute, (B) 5 minutes, (C) 10 minutes, (D) 30 minutes, and (E) 50 minutes (Magnification 100×).

\section{Results}

Control tests showed that protoscolices previously treated with warm water were functionally dead; they failed to reduce methylene blue at any of the two dye concentrations used and they permanently retained the blue color (Figs. 1 and 2). In contrast, untreated protoscolices reduced the dye effectively and eventually lost the blue color (Figs. 3 and 4). Following initial exposure to $1 \%$ methylene blue, normal protoscolices acquired the dye progressively over a period of 1 to 50 minutes (Fig. 5A-E). By 1-10 minutes (Fig. 5A-C), there was complete reduction of the dye as judged by complete disappearance of the blue color. This is followed by progressive influx of the dye which protoscolices failed to reduce (Fig. 5D and $\mathrm{E}$ ) accompanied by simultaneous depletion of the dye from around protoscolices. A notable result is that a clear distinction between viable (Fig. 5A) and dead protoscolices (Fig. 6) could be made within 1 minute following exposure to the dye. The Figure 7 shows normal invaginated protoscolices with rostellar hooks and calcareous corpuscles. 


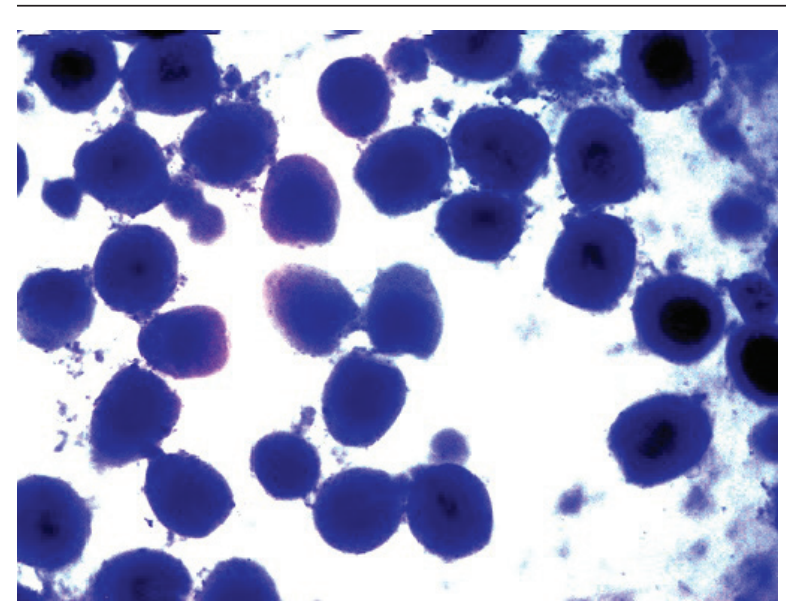

Fig. 6. Uptake of methylene blue by dead protoscolices after 1 minute of exposure (Magnification 100×).

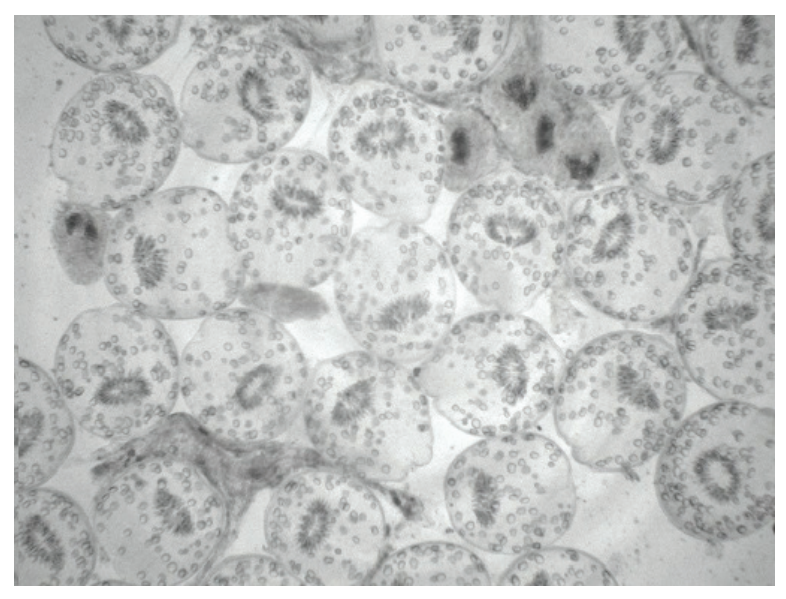

Fig. 7. Normal protoscolices (Magnification 100×).

\section{Discussion}

Hydatid cysts infect various species of animals as intermediate hosts with inherent differences, including prevalence and susceptibility to infection, organ disposition, or the degree of fertility of the cysts that develop in these animals. Validation of an effective method for assessment of viability of protoscolices may lead to a better understanding of the relative importance of different animal species in parasite transmission and may as well be useful in experimental studies, such as drug tests, in vitro parasite culture or immunological investigations.

Methylene blue is a redox indicator that loses its color in the absence of oxygen. According to Fioravanti and Saz (1980), parasitic helminths in general have the potential to effectively consume oxygen particularly at high pO2. In this respect, Farhan et al. (1959) found that protoscolices from hydatid cysts are able to carry on predominantly aerobic metabolism in vivo. Under an oxygen tension of atmospheric air (Agosin et al., 1957), the scolices consume oxygen at a rapid rate (Qo2 $=2$ ) with an overall RQ of 0.88 . The present findings suggest that methylene blue, by virtue of its chemical nature as a redox indicator; can be used effectively for assessment of viability of protoscolices. This was validated by control tests involving induced thermal death and the subsequent exposure of protoscolices to the dye. Exposure of protoscolices to the dye over a period of 50 minutes indicated that the distinction between dead and alive protoscolices, as judged by acquisition and loss/retaining of color, can be made within 1 minute. Hazratitappe et al. (2010) examined life signs of protoscolices treated with methylene blue at 2, 4, 8, and 16 minutes. They concluded that the dye has no killing effect on protoscolices up to this time. This is consistent with the results of the present study that protoscolices are effectively capable of reducing the dye at least up to 10 minutes (Fig. 5A-C). Whether the progressive influx of the dye in protoscolices after this time (Fig. 5D and E) and its accumulation in the oxidized form is conducive to oxygen toxicity and ultimate death remains to be defined.

A number of studies (Casado et al., 1986; Elissondo et al., 2004; Younes et al., 2011; Ismail and Saad, 2017) used protoscolex muscular movements, flame cell activity, morphological perfectness of the whole body, and evagination as criteria for assessment of viability of protoscolices applying eosin or methylene blue at a concentration of $0.1 \%$. The present study showed that increasing the concentration of methylene blue to $1 \%$ was associated with remarkable enhancement of contractility, sucker movement, and evagination of protoscolices. The application of this dye at a high concentration, therefore, may provide a better approach for assessment of protoscolex viability in studies that adopt these criteria.

\section{Conclusion}

It is proposed that the methylene blue can be used effectively for assessment of viability of protoscolices. Results, however, should be interpreted with caution; reading the test after 10 minutes would be misleading giving a false result.

\section{Acknowledgments}

This work was supported by a grant from the Deanship of Scientific Research, University of Khartoum.

\section{Conflict of interest}

The authors declare that there is no conflict of interest.

\section{References}

Agosin, M., Von Brand, T., Rivera, G.F. and McMahon, P. 1957. Studies on the metabolism of Echinococcus granulosus. I. General chemical composition and respiratory reactions. Exp. Parasitol. 6(1), 37-51.

Casado, N., Rodriguez-Caabeiro, F. and Hernandez, S. 1986. In vitro survival of Echinococcus granulosus protoscolices in several media, at $42^{\circ} \mathrm{C}$ and $37^{\circ} \mathrm{C}$. Z. Parasitenkd. 72, 273-278. 
Elissondo, M.C., Dopchiz, M.C., Brasesco, M. and Denegri, G. 2004. Echinococcus granulosus: first report of microcysts formation from protoscolices of cattle origin using the in vitro vesicular culture technique. Parasite 11, 415-418.

Elmahdi, I.E., Ali, Q.M., Magzoub, M.M.A., Ibrahim, A.M., Saad, M.B. and Romig, T. 2004. Cystic echinococcosis of livestock and humans in Central Sudan. Ann. Trop. Med. Parasitol. 98(5), 473-479.

Esfandiari, B. and Youssef, M.R. 2010. Comparison of eosin and trypan blue staining in viability of hydatid cyst protoscolices. Glob. Vet. 4(5), 456-458.

Farhan, I., Schwabe, C.W. and Zobel, C.R. 1959. Host-Parasite Relationships in Echinococcosis III. Relation of environmental oxygen tension to the metabolism of hydatid scolices. Am. J. Trop. Med. Hyg. 8(4), 473-478.

Fioravanti, C.F. and Saz, H.J. 1980. Energy metabolism of adult Hymenolepis diminuta. In Biology of the tapeworm Hymenolepis diminuta, Ed., Arai, H.P. Cambridge, MA: Academic Press, pp: 463-504.

Hazratitappe, K.H., Mousavi, S.J., Barazesh, A. and Asadi, A. 2010. In Vitro study of scolicidal effect of methylene blue in hydatid cyst. Iran. South Med. J. 13(2), 123-128.

Ismail, E.I.F. and Saad, M.B.E. 2017. In vivo and in vitro survival rates of protoscolices kept at different constant temperatures. Open J. Epidemiol. 7, 124130 .
Jeffs, S.A., Hurd, H., Allen, J.T. and Arme, C. 1987. Kinetics of molecular transfer across the tegument of protoscolices and hydatid cysts of Echinococcus granulosus and the relevance of these studies to drug targeting. In Helminth Zoonoses, Eds., Geerts, S., Kumar, V. and Brandt, J. Dordrecht, The Netherlands: Springer, pp: 37-43.

Miman, O., Aycan, O.M., Aydin, C. and Atambay, M. 2010. What should be the concentration of eosin to qualification of ideal staining for viability determination on hydatid cyst? Turk. Hij. Den. Biyol. Derg. 67(1), 21-26.

Moazeni, M. and Alipour-Chaharmahali, M.R. 2011. Echinococcus granulosus: in vitro effectiveness of warm water on protoscolices. Exp. Parasitol. $127(1), 14-17$.

Osman, O.M. and Abdalla, H.S. 2013. Prevalence of Echinococcus granulosus in stray dogs and hydatidosis in camels in Tambool Area, Gezira State, Sudan. Sudan. J. Vet. Res. 28, 15-18.

Saad, M.B. and Magzoub, M. 1986. Echinococcus granulosus infection in dogs in Tambool, Sudan. J. Helminthol. 60(4), 299-300.

Younes, D.A., Taher, G.A. and Ibraheim, Z. 2011. In vitro effects of some herbs used in Egyptian traditional medicine on viability of protoscolices of hydatid cysts. Korean J. Parasitol. 49(3), 255-263. 